\title{
Abstraction and Summarization of Meaning in Natural Language Processing
}

\section{Farida Huseynova}

Azerbaijan State Oil and Industry University, Baku, Azerbaijan

\begin{abstract}
Today's natural language processing system cause loss of information due to the approximation processes. The applied methods, based on Aristotle's binary logic usually cannot take the semantics into account in processing a language.In this paper, we tried to use an approach to analyze a set of terms given in a natural language and overcome some problems of processing.
\end{abstract}

Keywords- Semantic meaning, human, linguistic, beauty, machine understanding, lexicalization, content determination.

\section{INTRODUCTION}

The integral part of natural language systems are lexicons. However, understanding of some lexical designsin natural language becomes difficult.The understanding of semantic meaning of information handled automatically by machines has always been a difficult problem. For instance, humans always attempted to extract knowledge from large amounts of data or to interpret the functioning of complex systems and they were motivated to provide researches in the field.Zadeh [1] pointed out that humans have many remarkable abilities; one of them is the main ability "to converse, communicate, reason and make rational decisions in an environment of imprecision, uncertainty, incompleteness of information and partiality of truth."

The main goal of the paper is to explore the nature and computational use of meaning representations for word concepts in the context of a natural language understanding system.

Fuzzy set-based methods can be useful in such perspectives for their capacity to process linguistic information through the interface, they provide between numerical and symbolic values, and also for their intrinsic ability to reduce complexity by providing a synthesis of individual elements [2]. They enable users to have more friendly interactions with machines than many other methods of computational intelligence. It's not an easy task teaching machines to understand how we communicate, because the nature of the human language makes NLP difficult.

\section{OBJECTIVES}

Theprocessing of a semantic meaning has notwithstanding been strongly questioned and methods to improve it have been proposed.Comprehensively understanding the human language requires understanding both the words and how the concepts are connected to deliver the intended message. While humans can easily master a language, the ambiguity and imprecise characteristics of the natural languages create difficulties for NLP. A growing interest has been expressed more recently for fuzzy linguistic summaries that provide a textual description of numerical data. They have been introduced decades ago and are more and more studied because of the nowadays difficulty to grasp efficiently all available digital information. Interpretability of fuzzy models is a very complex criterion, difficult to define precisely, partly subjective and depending on the context of utilization.Textual representation of information can be more efficient than graphical ones in several cases. For instance, the data can be described in high dimensional domains, possibly and generally speaking hard to show graphically, in which case the linguistic summary is an interesting alternative [3].

As remarked in [4], the interpretability of semantic meaning of sentences has not been much studied by fuzzy rule-based systems. The understanding of a semantic meaning of a sentence in the text depends on its linguistic expression, and the linguistic variables attached to the attributes and to the fuzzy quantifier. The linguistic variables are usually defined by the user, both regarding the linguistic labels and the membership functions. In this case they can be considered as improving interpretability, since they somehow personalise the summaries to the user 
preference and his subjective perception of the attributes. However, such variables may be inappropriate with respect to the data structure. Automatic methods to extract a partition from the data can also be considered $[5,6]$.

In thesaurus dictionary the word beautyhas so many linguistic variables as charm, fascination, glamor, enchantment, allure, loveliness, etc. What is real beauty? Why does it have so many synonyms? The so- called universal attitude toward beauty was the theme of discussion for during the almost two million years of the Pleistocene.The criteria for beauty don't really apply today.

Jalal ad-Din Muhammad Rumi a poet, in the 13th century, described beauty as "The very center of your heart is where life begins - the most beautiful place on earth". $\mathrm{He}$ explained it as a reflection on nature's beauty, a song, a dance, an idea, a feeling... anything that makes us human, everything we're able to see with our eyes open or shut.

The things that we call beautiful are so different, because the understanding of beauty is provided by a perceptual experience of pleasure or satisfaction. There are the only ways of perceiving, besides the culture and language shape your perception of beauty. Beauty is anything that makes one feel good about a pleasing thing.We find beauty in something done well. These can be a combination of qualities, such as shape, color, or form which pleases the aesthetic senses, especially the sight. This brief list includes human beings, natural landforms, works of art and skilled human actions. The experience of beauty, with its emotional intensity and pleasure, belongs to our evolved human psychology, therefore, in the below mentioned sentences the words meaning beauty can be substituted by other linguistic variables according to our values and cultures.

There are separate words for beautyin different languages. In English the words magnificent, wonderful, lavish, (noble, attractive, good-looking express beauty too. We often think the way we experience the world must be the way it is. People of different cultures, ages, and genders use the language differently to express beauty.

We are interested if the beauty could be measured, and how the degreeof beauty is described in different situations by our senses? Brain responses to beauty differently as brains responds to beauty by linking vision and pleasure differently. Our judgments have reflective association to it too.Using different strong adjectives and adverbs helps to describeour different senses more clearly in different situations. In the sentences;

“Oh. Look at this pretty girl!"or “Oh. Look at this beautiful, (charming, nice) girl!
"Oh. What a handsome man he is"or "What a noble (lavish, attractive, good-looking) man he is."

"What an awesome smell your perfume has!" or "What a magnificent (wonderful, marvelous) smell your perfume has!"

“Wow! What a delicious dish!"or "Wow! What an adorable (lovely, adorable, magnificent, pleasant, tasty, mouthwatering, appetizing) dish!"

"This song is amazing!" or "This song is marvelous (magnificent, astonishing, magnificent, lovely!)"

Speakers usually choose a convenient unit to express beauty with different lexical units. As an illustration, it seems normal to declare "This song is lovely"

"Wow! What a delicious dish!" but odd to say "Wow! What a tasty, dish!". Once the linguistic variables are chosen, the values commonly used are often lends more weight to the meaning of a sentence.Wecan extend and intensify the creation and enjoyment of pleasure by different linguistic variables in different sentences. Isn't that exhaustively cultural? It's deep in our minds to make the ideas beautiful, to have them exert a kind of magnetism into words and express thepleasure simply looking atthe beauty. For us moderns, virtuoso technique is used to create imaginary worlds by the help of words and express our intense emotions.

It's a gift handed down from the intelligent skills and rich emotional lives of our most ancient ancestors. Our powerful reaction to images, to the expression of emotion in beauty can be expressed differently. For instance, one would rather say "Wow! What a mouthwatering dish!"This intensifies the semantic meaning" than "Wow! What an appetizing dish!”

It must be underlined that this appreciation is highly dependent on the context in which it is produced: it can happen that a preciseness of emotional intensity and pleasureis required, e.g. for certain events or auctions. Lastly, the word selection makes it possible to define the level of accuracy of statements. For expression of an emotion, it can be an adverb as "exactly", "approximately", "nearly", "roughly", "around". For instance, instead of saying "“"This song is amazing!" one would rather say "“This song is marvelous magnificent, astonishing", adding the adverb to indicate that this song expresses the beauty more exactly than the previous sentence. An implementation of these principles is proposed in $[5,6]$ to generate relevant linguistic expressions.

It is evident that there is a need for machines to be able to generate language rather than work only on understanding natural language that humans have uttered. Sometimes, the 
computer may fail to understand the meaning of a sentence well, leading to obscure results.

Though human involvement in lexicalization is noticed as a distinctive factor, it has its own disadvantages. Humans have different lexical preferences. With limited human involvement, lexicalization can be biased towards lexical preferences of those who are involved in evaluation. It is important to enhance the functionality of machine understanding for recognizing and understanding the human language.

\section{METHODS}

The processing of a meaning of sentences is not only based on the interpretability of each of its individual sentences but also on their interpretability as a whole. In this section, different aspectsof this global interpretability are explored. In order to aid computers to understand the human's natural language some important steps must be taken into consideration.

First, the property of consistency of the sentences isdetailed, then various methods to detect and remove redundancy are presented; finally, differenttechniques of information enhancement are introduced, dedicated to the creation of knowledgethrough links between the sentences.

It must be noted that theinterpretability presented here is related to ;

Content Determination is responsible for selecting information needed to becommunicated through generated text.

Document Structuring manages the structure of the information selected fromcontent determination.

Lexicalization operates on what words, terms and concepts need to be includedin the text.

Referring ExpressionGeneration is the process of determining the way thatentities must be referred within generated text.

Aggregation operation can be executed to structure and order the sentence structures to build a meaningful sentences.

Linguistic and Structure Realization is accountable for producing final surfacetext and presenting it based on the requirements. In this discussion we willrefer to this as to a surface realization which includes both aspects of realization.
Document planning

Content determination

Document structuring

Microplanning

Lexicalization

Aggregation

Realization

Referring expression generation

Linguistic

realization

Structure realization

Selecting the content plays a crucial role in NL process. Content determination

arises the issue which stops generalizing the lexicalization of the system even within the same domain being considered.

There are four aspects that need to be focused on during content determination:

- selecting data based on significance,

- summarizing data, so that important information is always included,

- include information derived through inference,

- customizing data based on the end-user needs.

In most current approaches, content determination is carried out by various methods where some of them have roots in early traditional approaches.

\section{CONCLUSION}

For making progress in conveying relevant and sufficient information of understanding the semantics in NLP, it is necessary to understand the existence of uncertainty in natural language. The vagueness, imprecision with some well-known applications of Google Search Engine, Google Translator and MIT Start were exemplified in the paper through real world examples for illustratingthe existence of the intelligence gap using well known natural language processing.

The interpretation capabilities of a language-understanding system depending on the semantic theory must be improved and fully accepted satisfactory in all respects. In our paper,the examples are chosen carefully to illustrate and demonstrate the applications of natural language processing environment for every reader.We think, accuracy of content extraction in natural language is necessary for overcoming theintelligence gap in such a simple manner in processing of vagueness in semantics 
[1] Aliev R.A., Fazlollahi B., Aliyev R.R..: Soft Computing and its Applications in Business and Economics, SpringerVerlag Berlin Heidelberg (2004).

[2] Zadeh L.A.: Test-score semantics for natural languages and meaning representation via PRUF, Emprical Semantics. Rieger B (eds) Germany: Brockmyer, pp 198-211, (1981)

[3] J. Yu, E. Reiter, J. Hunter, S. Sripada, SumTime-Turbine: A Knowledge-Based System to Communicate Gas Turbine Time-Series Data, in: Developments in Applied Artificial Intelligence, 2003, pp. 379-384.

[4] J. Kacprzyk, S. Zadrozny, Comprehensiveness and interpretability of linguistic data summaries: A natural language focused perspective, in: Proc. of IEEE SSCI CIHLI'13, 2013, pp. 33-40.

[5] S. Guillaume, B. Charnomordic, Generating an Interpretable Family of Fuzzy Partitions From Data, IEEE Trans.on Fuzzy Systems 12 (3) (2004) 324-335.

[6] L. Danlos, F. Meunier, V. Combet, EasyText: an Operational NLG System, in: Proc. of ENLG'11, 2011, pp.139-144.

[7] G. Moyse, M.-J. Lesot, B. Bouchon-Meunier, Mathematical morphology tools to evaluate periodic linguistic summaries, in: Proc. of FQAS'13, 2013, pp. 257-268.

[8] L. Danlos, F. Meunier, V. Combet, EasyText: an Operational NLG System, in: Proc. of ENLG'11, 2011, pp. 139-144. 